\title{
NASOPHARYNGEAL SPECIMEN COLLECTION FROM SUSPECTED COVID-19 CASES, MAKING THE PROCEDURE SAFE
}

\author{
Sohail Aslam, Syed Muhammad Asad Shabbir Bukhari, Adnan Asghar*, Naeem Riaz ${ }^{* *}$, Iftekhar Yousaf, Sana Sadiq***, \\ Muhammad Fahd Waseem \\ Pakistan Naval Ship Shifa, Karachi Pakistan, *Pakistan Naval Ship Rahat, Karachi Pakistan, **Pakistan Naval Ship Hafeez, Karachi Pakistan, \\ ***Niazi Medical and Dental College, Sargodha Pakistan
}

\begin{abstract}
Objective: To comparison was made between standard (control group) nasopharyngeal sample collection technique for RTPCR and modified technique and the outcome was compared in terms of the proportion of positive results of Rt-PCR tests.

Study Design: Double blinded randomized clinical trial.

Place and Duration of Study: Pakistan Naval Ship Shifa Hospital Karachi Pakistan, from June and July 2020.

Methodology: This study was a newly developed modified technique for nasopharyngeal sampling for RT-PCR tests of COVID-19 suspects. Target population included all patients who developed COVID-19 related symptoms and/or also had history of recent travel or closed contact with Covid-19 patients. Total 1500 nasopharyngeal PCR tests were done by a team of trained technicians. Systemetic probability sampling technique was utilized. Subjects were divided into two groups by using even and odd serial numbers. Proportion of positive test results were compared between two groups by using chi square test. Results: Results were collected for 3000 nasopharyngeal swab sample for RT-PCR testing. Mean age was $31.68 \pm 11.89$ years. In study group with modified technique, 470 tests were found positive for a total of 1500 samples while only 297 out of 1500 samples were detected positive in control group with standard technique. Chi square test applied to assess the difference between this proportion and it proved that the difference was highly statistically significant $(p$-value $<0.00)$.

Conclusion: we interpret that modified samples collection technique is relatively safe for sample collector of Covid-19 PCR which has got potential benefits to get more genuine results of these samples
\end{abstract}

Keywords: COVID-19, Nasopharyngeal sampling, safe procedure.

How to Cite This Article: Aslam S, Bukhari SMAS, Asghar A, Riaz N, Yousaf I, Sadiq S, Waseem MF. Nasopharyngeal Specimen Collection from Suspected COVID-19 Cases, Making the Procedure Safe. Pak Armed Forces Med J 2021; 71 (Suppl-3): S432-436. Doi: https://doi.org/10.51253/pafmj.v1i1.7925

This is an Open Access article distributed under the terms of the Creative Commons Attribution License (https://creativecommons.org/licenses/by-nc/4.0/), which permits unrestricted use, distribution, and reproduction in any medium, provided the original work is properly cited.

\section{INTRODUCTION}

A pathogenic viral infection, COVID-19, has emerged as a new global public health crisis.1,2 COVID-19 disease (COVID-19) caused by SARS-COV-2 has affected many cities of Pakistan too since February 2020. Therefore, a need was felt to expand the testing capabilities for this disease in order to find positive cases in the country for putting them in isolation either, or to provide effective treatment. Considering patients' convenience, sensitivity and accuracy of the test, nasopharyngeal specimen for nuclieic acid estimation of COVID-19 disease is most commonly used everywhere now a days. For identification of viral nuclieic acid, reverse transcriptase polymerase chain reaction (RTPCR) a hallmark of nasopharyngeal swab test is used. This test, however, is considered very risky for sample collectors, as there are high chances that patient/suspect may sneeze, cough, vomit or breathe hard during the procedure, thus generating large number of aerosols or droplet, putting sample collectors at a very high risk of cross infection. Therefore, sample collectors are

Correspondence: Dr Syed Muhammad Asad Shabbir Bukhari, Classified ENT Specialist, PNS Shifa, Karachi Pakistan liable to get inappropriate samples as risk of getting COVID-19 cross infection puts them under lots of psychological stress and fear. This practice has affected the sensitivity of nasopharyngeal swab sampling adversely and lead to high percentage of false negative test results. Although the sensitivity of nasopharyngeal swab for PCR ranges between $63-78 \%$, but its specificity is very high and appropriate technique should be used to get the best results. Although, a closed contact between sample collector and COVID-19 suspect while doing nasopharyngeal swab is must but few modifications in standard practiced technique of sample collection could decrease amount of aerosol/droplet generation and reduced fear and stress among sample collectors which could definitely improve the accuracy of nasopharyngeal swab technique. These modifications were developed by a team of Otorhinolaryngologists (authors), specialists in dealing diseases of nasopharyngeal area. This modified technique was expected to collect precise sample with better yield of positive results.

In this study comparison was made between standard (control group) nasopharyngeal sample coll- 
ection technique for RT-PCR and modified technique and the outcome was compared in terms of the proportion of positive results of RT-PCR tests.

\section{METHODOLOGY}

This was a double blinded randomized clinical trial of a newly developed modified technique for nasopharyngeal sampling for RT-PCR tests of COVID19 suspects. The study was carried out at Pakistan Naval Ship shifa Hospital Karachi, from June and July 2020.

Inclusion Criteria: Arget population included all patients who developed COVID-19 related symptoms and/or also had history of recent travel or closed contact with COVID-19 patients. Our accessible population included all COVID-19 suspects undergoing diagnostic tests in PNS Shifa hospital Karachi. Out of accessible population, our sample was 3000 nasopharyngeal PCR tests, approximately 100 tests each day in the month of June and July 2020, for COVID-19 suspects. Informed consent was obtained from all patients before including them in the study.

Exclusion Criteria: Patients not cooperating during sampling as per steps of technique were excluded from the study. Similarly those already diagnosed positive COVID were excluded from study.

Total 1500 nasopharyngeal PCR tests were done by a team of trained technicians who used standard nasal swab technique which is being practiced worldwide and other 1500 tests were done by another team of technicians who used our proposed modified technique. Each team comprised of 5 members. Systematic probability sampling technique was utilized. Subjects were divided into two groups by using even and odd serial numbers. The individual at main reception allotting serial number to all patients was instructed to allocate group to the patient. Even numbers were allotted to the suspects who were tested using modified technique while odd numbers were allotted to the suspects tested by using standard nasopharyngeal swab sampling technique. Neither authors (data analysts) nor the patients were aware of group allocation. Results of PCR tests of all subjects enrolled in study were obtained from laboratory.

Data was analyzed using SPSS-22. Proportion of positive test results were compared between two groups by using chi square test. Potential confounding variables of age and gender were also analyzed between two groups. Numerical variable of age was analyzed by independent sample t-test while categorical data of gender by chi square test. $p$-value was set as $<0.05$ for significance of difference.

\section{Standrad Technique for Nasopharyngeal Sample Collection}

These samples are taken by registered technician using viral transport medium (VTM). Before sampling, suspects and attendant are informed about the procedure. Pictures are also shown to the suspects about the procedure of nasopharyngeal sampling swab which ensures patient's cooperation during the procedure. Standard Personal protection equipment (PPE) is used by sample collectors which also includes N-95 mask and face shield.Written and informed consent is taken from each patient before procedure.

Polyester flexible, mini tips swabs are passed through the suspect's nostril, until the nasopharynx is reached, left in place for 15-20 seconds to absorb secretions, and then slowly removed by rotating fashion. The swabs then are placed in viral transport medium. This is shown in Figure-1.

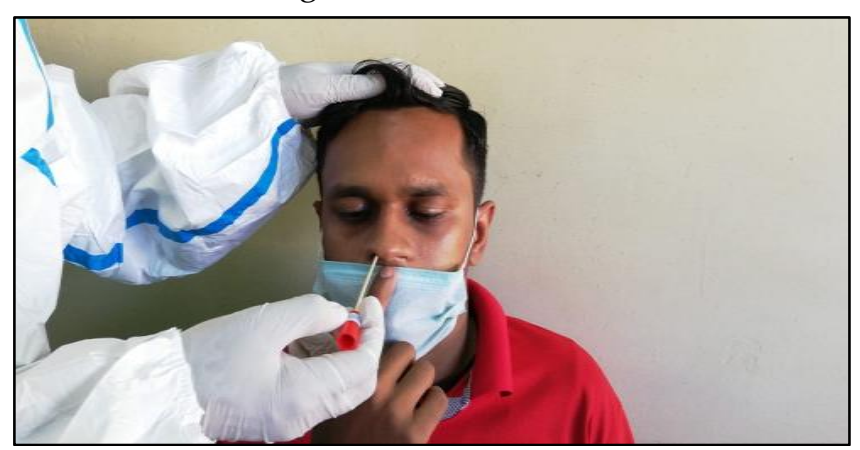

Figure-1: Standrad technique for nasopharyngeal sample collection.

\section{Modified Technique for Nasopharyngeal Sample Collection}

In this technique few modifications were introduced which could reduce the stress and fear among sample collectors. Moreover, it might reduce the element of false negative results. The steps included are: Wearing standard PPE which includes N-95 mask and face shield.

\section{Self-Introduction}

Written/informed consent.

Introduction about the procedure and explanation by showing pictures of nasopharyngeal swab technique to the suspect.Ask from suspect about patency of nose and use more patent nostril for sampling to reduce nasal irritation.

Cover the mouth of suspect with surgical mask and just expose the nostrils, to reduce aerosol gene- 
ration if patient starts coughing or harsh breathing during the procedure.

Avoid hyper extension of neck (to keep patient face \& head in natural position) to avoid inadvertent damage to the nasal roof. Use swab stick with wooden shaft which is easy to insert and without being kinked, which mostly occur with use of plastic swab sticks and increases chances of sneezing and patient's agony.

Keep swab sticks direction medial and inferior in nostril while approaching nasopharynx which definitely will reduce nasal irritation. Keep stick end in nasopharynx for 15- 30 seconds and then remove it rotating fashion. Tell suspect to place a figure on upper lip while being tested which can reduce chances of accidental bout of sneezing or coughing. Sample collector should not face the suspect directly. He should keep his face 45 degree of suspect's right side while inserting swab stick in nostril. This act might further reduce direct contact with aerosol generated by suspect.

Moreover, sample collector should move 6 feet away from suspect as soon as he inserts swab stick in nasopharynx. Wait for 15-20 seconds and then move back closer to the suspect for removal of swab stick. This step of procedure is imperative to reduce exposure of sample collector from aersol generated by suspect. This step also could play pivotal role in reducing sample collector's fear (figures-2 \& 3).

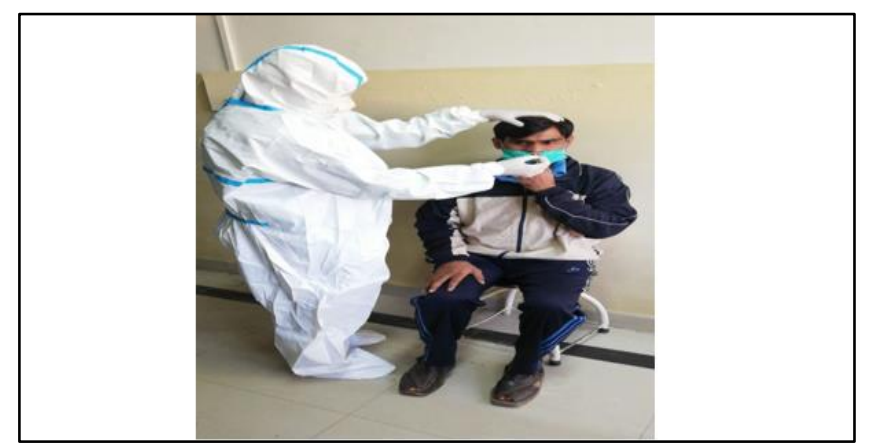

Figure-2: Modified technique for nasopharyngeal sample collection-step 1.

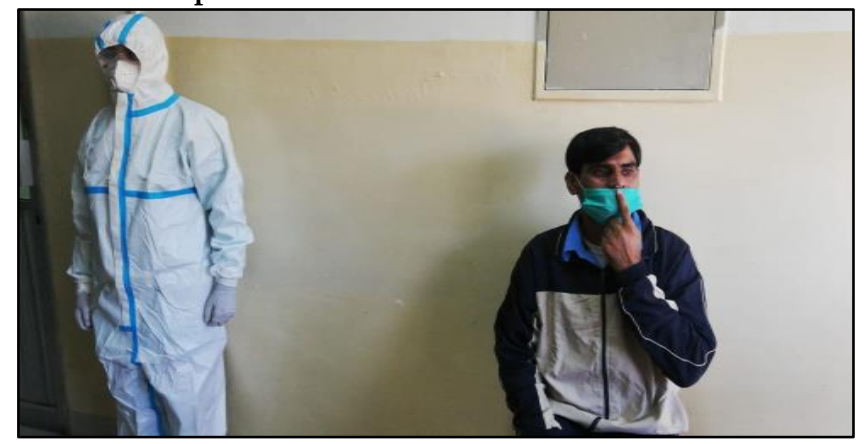

Figure-3: Modified technique for nasopharyngeal sample collection-step 2.

\section{RESULTS}

Results were collected for 3000 nasopharyngeal swab sample for RT-PCR testing. Mean age was 31.68 (Standard deviation 11.89). Considering age as a potential confounding variable, mean ages of two groups were compared by applying t-test. Calculated $p$-value proved that the difference was not statistically significant, $p$-value 0.074 (95\% confidence interval of difference-0.421, 1.283). Out of these 3000 COVID-19 suspects 2681 were male and 319 were females. The difference of gender distribution between two groups was also not statistically significant ( $p$-value 0.678 by chisquare test). In study group with modified technique, 470 tests were found positive for a total of 1500 samples while only 297 out of 1500 samples were detected positive in control group with standard technique (Figure-4).

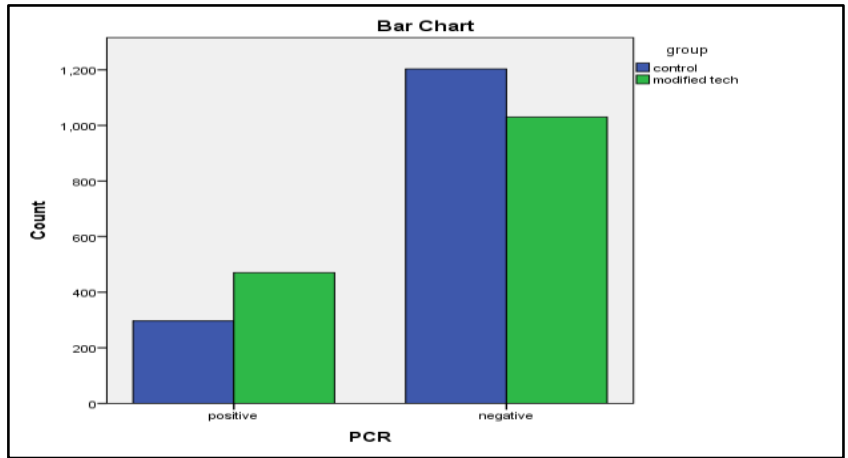

Figure-4: Comparison of proportion of positive tests between two groups.

Chi square test applied to assess the difference between this proportion and it proved that the difference was highly statistically significant (Pearson Chi square test $p$-value $<0.00$ ) (Table).

Table: Statistical tests and $p$-values of gender, age and PCR test.

\begin{tabular}{|c|c|c|c|c|}
\hline Variables & Control & Modified & Test & $p$-value \\
\hline Male & 1344 & 1337 & \multirow{2}{*}{ Chi-square } & \multirow{2}{*}{0.678} \\
\hline Female & 156 & 163 & & \\
\hline PCR+ & 297 & 470 & \multirow{2}{*}{ Chi-square } & \multirow{2}{*}{0.000} \\
\hline PCR- & 1203 & 1030 & & \\
\hline Age & Mean 31.9 & Mean 31.4 & t-test & 0.321 \\
\hline
\end{tabular}

\section{DISCUSSION}

COVID-19 is a viral disease, therefore it does not usually produce purulent secretions. The sample from nasopharynx or oropharynx is usually collected for the PCR tests. Nasopharyngeal sample is considered to have more diagnostic yield than oropharyngeal sample. The anatomy of nose and nasopharynx is variable, therefore sampling becomes challenging but still it is 
easier compared to broncho-alveolar lavage. In order to diagnose COVID-19, nasopharyngeal swab for RTPCR is preferred technique considering patients, convenience and because of reason many hospital are not equipped with facilities related to broncho-alveolor lavage. ${ }^{3,4}$ The sensitivity of nasopharyngeal samples for RT-PCR may be affected if samples contain inadequate amount of viral RNA.5,6 This could be attributed to the assumption that sample collectors get inappropriate amount of viral RNA while collecting nasopharyngeal samples from COVID-19 suspects with fear and stress of getting cross infection.

In this study we introduced a modified technique with inclusion of few steps in nasopharyngeal sample collection technique to enhance its safety level and compared it with existing standard technique. This technique was finalized after detailed discussion between many senior otorhinolaryngologists (authors).

The results of this study clearly showed that modified technique is much better than standard technique based on more number of positive test results found using modified technique for COVID-19 nasopharyngeal samples.

Modified sampling technique for nasopharyngeal swab minimizes contact between sample collector and COVID-19 suspect and also reduces exposure to the aerosol generated by the patients, therefore reducing the risk of viral transmission. As COVID-19 pandemic in our country increased, the demand for nasopharyngeal RT-PCR swabs too was increased, so a need was felt to introduce a safer technique and we decided few modifications and included them in nasopharyngeal swab collection techniques, rather than looking for alternative sampling methods.

Moreover, in the current study, sample collectors in control groups were not satisfied with the traditional technique of nasopharyngeal sample collection for COVID-19 and remained anxious and guarded during the procedure as per responses given by them in the questionnaire. All had fear of getting cross infection during the procedure. Gan et al, observed serious occupational health risk to the health care professionals including sample collectors owing to their frequent exposure to infected individuals with COVID-19.7 To some extent, these concerns were also related to the miss information regarding COVID-19 pandemic leading to xenophobia among people all around the world. 8,9 Non availability of vaccine against COVID-19 so far could be a contributing factor affecting sample collector confidence, performance and efficacy. How- ever, $80 \%$ of sample collectors in study group were not anxious while $20 \%$ remained neutral while responding to the questions in the questionnaire.

Past studies also has reported extra ordinary stress among health care providers during SARS and MERS epidemic due to high infection risk, under staffing, stigmatization, uncertainty and comprehensive support of highlighted during and after the outbreak. 10,11 Furthermore, thousands of health care workers and staff were infected or died in China, Spain, Italy, Turkey and other parts of the world during this pandemic and such reports were fair enough to induce anxiety and fear among sample collectors. ${ }^{12-14}$

These findings necessitate development of safe measures and environment for health care providers and sample collectors to boost up their efficiency and performance. Availability of vaccines for COVID-19 is desperately awaited. Scientific challenges in this aspect are being studied these days. ${ }^{15}$ Studies regarding role of screened set of epitopes and use of nanotechnology are very helping in development of vaccine. ${ }^{16,17}$ Mark et al, studied safety, tolerability and immunogenicity data of mRNA vaccine. Their results supported further evaluation of this vaccine. ${ }^{18}$

We did this study in 3000 cases and divided them into two groups of 1500 individuals each. The aim of getting large number of cases/sample size was to find out better and more authentic results. However, we feel that modified nasopharyngeal sample collection should be replicated in other centers as well and should also be compared with traditional technique of nasopharyngeal sample collection in order to learn more about its pros and cons. Finally, we interpret that modified samples collection technique is relatively safe for sample collector of COVID-19 PCR which has got potential benefits to get more genuine results of these samples. This newer modified sample collection technique also reduces stress and anxiety among sample collectors, thus enhancing their efficacy and confidence while doing this procedure.

\section{CONLUSION}

we conclude that modified samples collection technique is better than conventional technique for sample collector of COVID-19 PCR which has got several profits to get more true positive results of these samples with more safety.

\section{ACKNOWLEDGEMENT}

We are thankful to our parents, teachers and friends. 


\section{LIMITATIONSOF STUDY}

The study is conducted in only one hospital.

\section{Conflict of Interest: None.}

\section{Authors' Contribution}

SA: Introduction and master of new technique, SMASB: Collection of data, AA: Data discussion, NR: Methodology, IY: Results, SS: Review of literature, MFW: Analysis of data.

\section{REFERENCES}

1. Shereen MA, Khan S, Kazmi A, Bashir N, Siddique R. COVID-19 infection: origin, transmission, and characteristics of human coronaviruses. J Adv Res 2020; 24(2): 91-98.

2. Singhal T. A review of coronavirus disease-2019 (COVID-19). The Ind J Pediat 2020: 1(1): 1-6.

3. Wolfel R, Corman VM, Guggermos W, Seilmaier M, Zange S, Muller MA, et al. Virological assessment of hospitalized patients with COVID-2019, Nature 2020; 581(7809): 465-469.

4. Yu C, Li L, Tuersun Y, Zhao X, Feng Q, Zhang T, Tay FR, Ma J. Oropharyngeal secretion as alternative for SARS-CoV-2 detection. J Dental Res 2020; 99(10): 1199-205.

5. Cheng MP, Papenburg J, Desjardins M, Kanjilal S, Cpuach C, Libman $M$, et al. Diagnostic testing for severe acute respiratory syndrome-related corona virus-2: a narrative review. Ann Intern Med 2020; 172(11): 726-734.

6. Loopi G, Simundic AM, Plebani M. Potential preanalytical and analytical vulnerabilities in the laboratory diagnosis of coronavirus disease 2019 (COVID-19). Clin Chem Lab Med 2020; 58(7): 1070-1076.

7. Gan WH, Lim JW, Koh D. Preventing intra-hospital infection and transmission of COVID-19 in health careworkers. Saf Health Works 2020; 11(2): 241-243.
8. Lai CC, Shih TP, Ko WC, Tang HJ, Hseuh PR. Severe acute respiratory syndrome coronavirus 2 (SARS-CoV-2) and coronavirus disease-2019 (COVID-19): The epidemic and the challenges. Inter J Antimicribial Agent 2020; 55(3): 105924.

9. Shimizu K. 2019-nCoV, fake news, and racism. The lancet, Lancet Publishing Group 2020; 3(1): 685-686.

10. Lee SM, Kang WS, Cho A-R, Kim T, Park JK, Psychological impact of the 2015 MERS outbreak on hospital workers and quarantined hemodialysis patients. Comp Psych 2018; 87(2): 123-127.

11. Maunder R, Hunter J, Vincet L, Bennett J, Peladeau N, Leszcz M, et al. The immediate psychological and occupational impact of the 2003 SARS outbreak in a teaching hospital. Can Med Assoc J 2003; 168 (10): 1245-51.

12. Lai J, Ma S, Wang Y, Cai Z, Hu J, Wei N, et al. Factors associated with mental health outcomes among health care workers exposed to coronavirus disease 2019. J Am Med Assoc Network Open 2020; 3(3): e203976.

13. Chen $W$, Huang Y. To protect health care workers better, To save more lives with COVID-19. Anesth Analg 2020; 131(1): 97-101.

14. ERSOY A. The frontline of the COVID-19 pandemic: Healthcare workers. Turk J Inter Med 2020; 2(2): 31-32.

15. Mangalakumari J, Afkhami S, Fiona S, Matthew SM, Brian DL, Zhou X. Immunological considerations for COVID-19 vaccine strategies. Nat Rev Immunol 2020; 20(1): 615-632.

16. Ahmed SF, Quadeer AA, McKay MR. Preliminary identification of potential vaccine targets for the COVID-19 coronavirus (SARSCoV-2) based on SARS-CoV immunological studies. Virus 2020; 12(3): 254-260.

17. Shin MD, Shukla S, Chung YH. COVID-19 vaccine development and a potential nanomaterial path forward. Nat Nanotechnol 2020; 15(1): 646-655.

18. Mulligan MJ, Lyke KE, Kitchin N. Phase I/II study of COVID-19 RNA vaccine BNT162b1 in adults. Nature 2020; 586(4): 589-593. 